\title{
Research on fuzzy control of the vehicle's semi-active suspension
}

\author{
Zhengke Chen \\ Automotive Engineering institute, Jiangxi University of Technology, Nanchang 330098, China
}

Keywords: Fuzzy control; Semi-active suspension; Vehicle

\begin{abstract}
The suspension is one of assembly of the modern automobile, the general term of all the force transfer devices between a frame (or chassis and body integral construction) and axle (or wheel). Its main function is to transmit the vertical reaction (supporting force), longitudinal reaction (traction and braking force) and lateral reaction, which are generated by the pavement acting on the wheel, as well as the torque caused by these reactions to the frame (or chassis and body integral construction), thus easing the impact load that the uneven road transmits to the body and reducing the vibration of the bearing system caused by the impact load; at the same time the motion of the body and the wheel should be guided to determine the position of the wheel; when the vehicle is heeling or pitching, the suspension needs to control the posture of the body timely to guarantee the normal running of the vehicle. Therefore, the design of the vehicle's suspension system must fulfill the requirements of ride comfort and handling stability. This paper will establish a nonlinear mathematical model of the semi-active suspension system for four degrees of freedom $1 / 2$ vehicle. The fuzzy logic control method is applied to design the fuzzy controller with different adjustment factors, and then the control simulation software Matlbas/imulnik is used to implement computer simulation.
\end{abstract}

\section{Introduction}

At present, the vehicles in our country mainly use the passive suspension. The two degrees of freedom system model is shown in the first one in Fig. 1. The traditional passive suspension is composed of the spring and damper with general fixed parameters. The elastic characteristics of its spring and the damping features of its damper cannot be adjusted with the change of vehicle operating conditions. Besides, the various components do not consume energy from the outside when working, so it is called passive suspension. It has a simple structure and reliable performance, which has been basically mature after continuous improvement now.

With the further increase of vehicle speed, people put forward higher requirements on the ride comfort. In order to overcome the defects of passive suspension, people begin to seek a new suspension system that can meet the requirements of performance. This suspension system improves the performance of vehicle suspension system fundamentally and provides a brand new way. The second one in Fig. 1 is the two degrees of freedom active suspension system. Compared with the active suspension, the semi-active suspension system has no force generator. It adjusts the hydraulic resistance of the damper by inputting a small amount of control energy to improve the vibration characteristics of the suspension. The third one in Fig. 1 is the two degrees of freedom semi-active suspension system. The system is mainly composed of the elastic element and the adjustable damper. The adjustable damper is similar to that of the passive suspension in generating force, but its damping coefficient sends feedback signal based on the vertical acceleration of the body mass and 
the relative displacement of sprung mass and unsprung mass, and it changes transiently according to certain control rules, so the damping effect is close to full active suspension. But because the stiffness of the spring in the semi-active suspension is a certain value, its self-adaptive function is relatively inferior to that of full active suspension. The semi-active suspension can be divided into level adjustable damping and continuously adjustable damping.
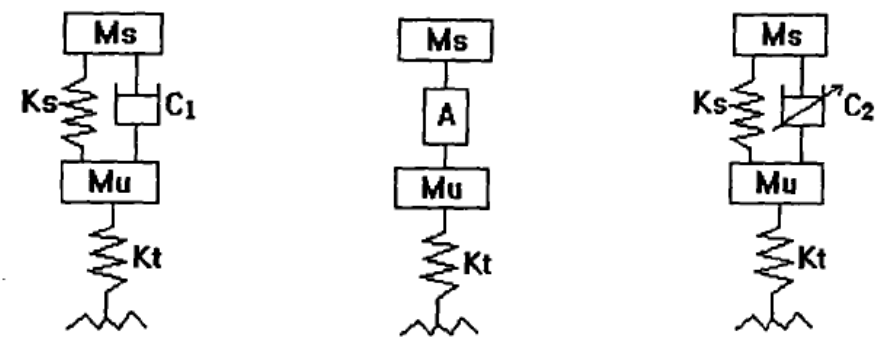

Fig. 1 three types of suspension models

\section{The establishment of nonlinear system model of the semi-active suspension}

Because the car is a complex vibration system which is often simplified in order to analyze and solve the problems. When the car is symmetrical to the longitudinal axis, the roughness functions of the right and left ruts are equal, so at this time what need to be considered about the car body are vertical vibration and pitch vibration, in which case the car can be simplified as a plane model of 4 degrees of freedom. The object of this study is the vibration system model of four degrees of freedom vehicle with semi-active suspension (Fig. 2). Here, the suspension system is treated as a nonlinear system composed of a nonlinear spring and a nonlinear damper. Because the tire damping is relatively small, it can be ignored, so the tire can be considered as a nonlinear spring. According to a large number of references and practical experience, the nonlinear function of the spring can be expressed by the following formula:

$$
F_{s}=\left.K_{s} \operatorname{sgn}(\Delta X) \Delta X\right|^{n}
$$

Among them, $\mathrm{F}$ is the damping force while $\mathrm{C}$ and $\mathrm{CC}$ are the tension and compression damping coefficients respectively. Usually $\frac{C_{i}}{C_{c}}=3^{\sim} 5$. Based on a large number of references and practical experiences, the value is 3 . 


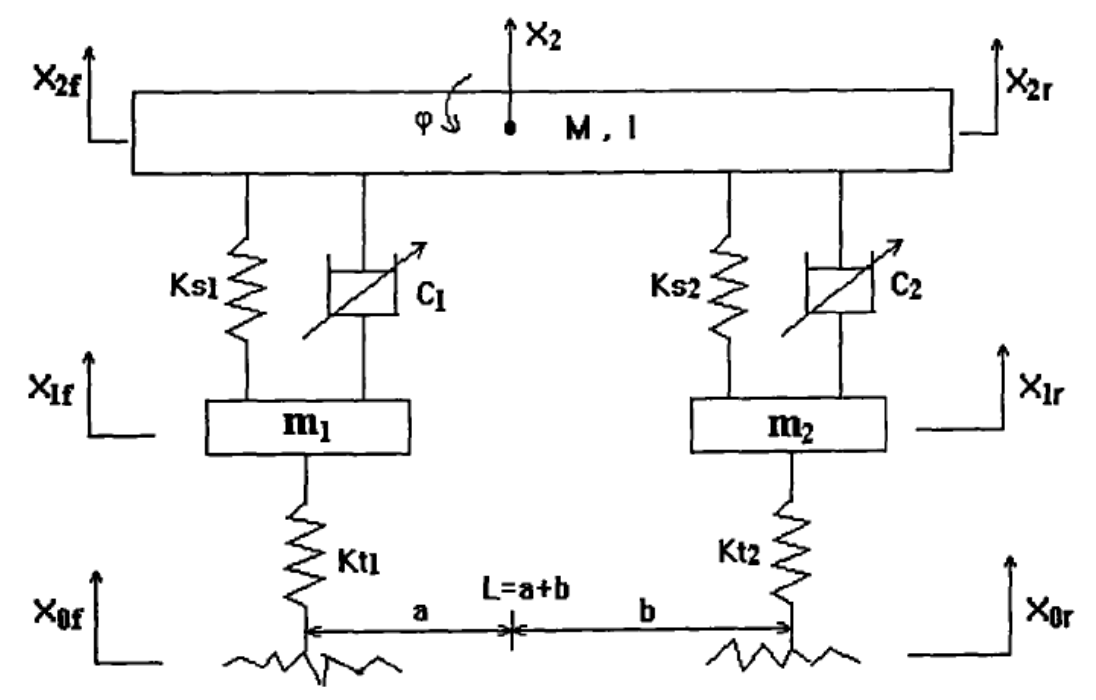

Fig. 2 the vibration model of the vehicle

The method uses control damping, so in terms of the model of suspension, the semi active suspension and the passive suspension are basically the same, but the damping value of the former equation is adjustable variable while the damping value of the latter equation is constant. Thus, we obtain the mathematics models of the nonlinear system of semi-active suspension. Based on this model, the software Matlba Simulnik can be used to design the control model of nonlinear system for semi-active suspension.

The road input $X_{0}=\left[\begin{array}{ll}X_{0 f} & X_{0 r}\end{array}\right]$ is random. The pavement is roughness. As the vehicle vibration inputs, it mainly adopts the pavement power spectral density to describe the statistical characteristics. It is recommend that the pavement power spectral density $\mathrm{n}()$ should use the following formula as the fitting expression:

$$
G_{q}(n)=G_{q\left(n_{0}\right)}\left(\frac{n}{n_{0}}\right)^{-W}
$$

In the formula:

$\mathrm{N}$--- spatial frequency. It is the reciprocal of wavelength, expressing the wave length in each meter;

W --- the frequency index, which is the slope of the diagonal in log-log coordinate. It determines the frequency structure of road spectrum. Usually $\mathrm{w}=2$.

\section{The fuzzy control semi-active suspension and its simulation research}

Fuzzy control is a computer numerical control based on fuzzy set theory, fuzzy linguistic variables and fuzzy logic inference. Before the existence of the automatic control technology, in the production process, people could only use manual control mode, whose procedure was first to observe the output of the object, then to make a decision according to the observation results, and finally to adjust the input manually. With the development of science and technology, people gradually use various measuring devices, controllers and actuators to achieve observation, decision making and adjustment of the object, so as to form the automatic control system. This is also known as the conventional negative feedback control system. 
When the founder of control theory Weiner studied the interaction between man and environment, he pointed out that "people perceive the surrounding world through the perception of sensory organs and adjust the obtained information in the brain and nervous system. Through proper storage, correction, induction and selection (processing), the information enters the effect organ to react in the outside world (output), but also acts on the central nervous system through some sensors as motion sensor terminals. It will integrate the newly-received information with the originally-stored information to influence and direct the future action". As Wiener described, take the driver's parking as an example. It is to obtain information continuously from the outside (object), then to store and process information, and to make the decision to react to the outside world (output), and finally to achieve the expected objectives.

The fuzzy controller is mainly composed of four parts. Fuzzification plays an important role in dealing with uncertain information. In the fuzzy control, the observed data are clear. The fuzzy operation is to map the measurement in the input space as fuzzy sets in the input domain. The input includes the external input reference and the output and state of the system input. The specific procedure is:

(1) The input is processed to become the input required by the fuzzy controller. The common case is calculation.

(2) The processed input is transformed in scale to make it in their respective domain.

(3) The transformed input implements fuzzy processing to make the originally accurate input into fuzzy one that is expressed by the corresponding fuzzy set.

Usually it is composed of database and fuzzy control rules. The database contains relevant parameters of fuzzy control rules and fuzzy data processing, including scaling transformation parameters, fuzzy spatial segmentation and the selection of membership function. Fuzzy control is a control method to imitate human, in which a set of rules described in language is used to express the knowledge of experts. Fuzzy inference plays an important role in the fuzzy controller, which can simulate human's inference ability based on fuzzy concept and whose reasoning process is based on the implication relation and inference rules in fuzzy logic. For a fuzzy controller with multiple input multiple output (M MO), whose rule base has the following form:

$$
R=\left\{R_{\text {umo }}^{1}, R_{\text {umo }}^{2}, \ldots \quad R_{\text {umo }}^{m}\right\}
$$

If $R_{\text {umo }}^{1}$ (x is $\mathrm{A} 1$ and... and $\mathrm{y} \mathrm{B} 1$ ), then $(\mathrm{Z} 1$ is $\mathrm{C} 11, \ldots . \mathrm{Zn}$ is $\mathrm{C} 1 \mathrm{q})$

The fuzzy controller plays a decisive role in fuzzy control system, so the design of the fuzzy controller in the fuzzy control system should contain the following contents:

(1) determine the input variables and output variables of the fuzzy controller (i.e. control);

(2) design the control rules of fuzzy controller;

(3) determine the fuzzy and non fuzzy (also called clear) method;

(4) select the domain of input variables and output variables of the fuzzy controller and determine the fuzzy controller.

\section{Conclusion}

The paper refers to the vehicle semi-active suspension system as the research object. It establishes the nonlinear system model of four degrees of freedom vehicle, adopts the fuzzy logic control strategy and designs fuzzy controller for different scaling factors; and it optimizes the design of 
fuzzy control rules using genetic algorithm. Respectively, simulation and real vehicle test are carried out. Through the above research work, the following conclusions can be drawn:

1. Based on the analysis of the performance of vehicle semi active suspension, the paper establishes a controlled model of nonlinear system for the semi-active suspension. The model considers the vertical vibration and pitch angle vibration of the vehicle and the effect of the front and rear wheels incentive on the vehicle vibration, which is suitable for vehicle dynamics research.

2 Control methods of fuzzy logic are used in the vehicle semi-active suspension system, which can solve the problems of uncertainty and nonlinear control relatively better to achieve a more ideal control effect.

3 The selection of fuzzy control rules is the key to the control method, and the selection of rules often relies on the experience of the experts, so it will have a certain impact on the control precision. Based on Genetic Algorithm, the fuzzy control system has better response than that of the control system of artificial design, and it is robust to changes in parameters, which can save the time for designing thee fuzzy controller.

4 The real vehicle test results are basically consistent with simulation results, showing that the mathematical model is correct and the control method is feasible. It also proves that the fuzzy controller based on genetic algorithm has a better control effect than the controller without the suspension system, thus improving the vehicle ride comfort.

To enable the semi-active suspension system to have superior performance, there are the following problems to consider.

1. The establishment of the semi active suspension system model has better consider the multi degrees of freedom and multi mass system model for the whole car and the acceleration of the sprung mass, body pitching, heeling, suspension deflection, wheel load and other performance indicators.

2. A more effective and quicker method to evaluate the performance of fuzzy control system can be found out so that it can better play its role of control.

3. As for the nonlinear characteristics of vehicle semi-active suspension, nonlinear, combining the genetic algorithm and fuzzy logic and neural network to make them compensate each other, a more effective control method should be designed so as to enable it to develop in the direction of automatic control and intelligent control.

\section{Acknowledgements}

This work was financially supported by the key subject building project (vehicle engineering) of Jiangxi University of Technology.

\section{References}

[1] Inagaki S, Kshiro I, Yamamoto M. Analysis on vehicle stability in critical cornering using phase-plane method[C]/International Symposium on Advanced Vehicle Control (1994: Tsukuba-shi, Japan). Proceedings of the International Symposium on Advanced Vehicle Control 1994. 1994.

[2] Du H, Sze K Y, Lam J. Semi-active Hœ control of vehicle suspension with magneto-rheological dampers[J]. Journal of Sound and Vibration, 2005, 283(3): 981-996. 
[3] Naab K N, Reichart G. Driver assistance systems for lateral and longitudinal vehicle guidance[C]//International Symposium on Advanced Vehicle Control (1994: Tsukuba-shi, Japan). Proceedings of the International Symposium on Advanced Vehicle Control 1994. 1994.

[4] Priyandoko G, Mailah M, Jamaluddin H. Vehicle active suspension system using skyhook adaptive neuro active force control[J]. Mechanical Systems and Signal Processing, 2009, 23(3): 855-868.

[5] Li H, Liu H, Gao H, et al. Reliable fuzzy control for active suspension systems with actuator delay and fault[J]. Fuzzy Systems, IEEE Transactions on, 2012, 20(2): 342-357.

[6] Khajavi M N, Abdollahi V. Comparison between optimized passive vehicle suspension system and semi active fuzzy logic controlled suspension system regarding ride and handling[C]//Proceedings of world academy of science, engineering and technology. 2007, 21: 57-61.

[7] Yagiz N, Hacioglu Y, Taskin Y. Fuzzy sliding-mode control of active suspensions[J]. Industrial Electronics, IEEE Transactions on, 2008, 55(11): 3883-3890.

[8] Yu M, Liao C R, Chen W M, et al. Study on MR semi-active suspension system and its road testing[J]. Journal of intelligent material systems and structures, 2006, 17(8-9): 801-806.

[9] Lin Y J, Lu Y Q, Padovan J. Fuzzy logic control of vehicle suspension systems[J]. International Journal of Vehicle Design, 1993, 14(5): 457-470.

[10]Eski I, Yıldırım Ş. Vibration control of vehicle active suspension system using a new robust neural network control system[J]. Simulation Modelling Practice and Theory, 2009, 17(5): 778-793. 